\title{
Experimental vortex breakdown topology in a cylinder with a free surface
}

\author{
Lo Jacono, D.; Nazarinia, M.; Brøns, Morten
}

Published in:

Physics of Fluids

Link to article, DOI:

$10.1063 / 1.3265718$

Publication date:

2009

Document Version

Publisher's PDF, also known as Version of record

Link back to DTU Orbit

Citation (APA):

Lo Jacono, D., Nazarinia, M., \& Brøns, M. (2009). Experimental vortex breakdown topology in a cylinder with a free surface. Physics of Fluids, 21(11), 111704. https://doi.org/10.1063/1.3265718

\section{General rights}

Copyright and moral rights for the publications made accessible in the public portal are retained by the authors and/or other copyright owners and it is a condition of accessing publications that users recognise and abide by the legal requirements associated with these rights.

- Users may download and print one copy of any publication from the public portal for the purpose of private study or research.

- You may not further distribute the material or use it for any profit-making activity or commercial gain

- You may freely distribute the URL identifying the publication in the public portal

If you believe that this document breaches copyright please contact us providing details, and we will remove access to the work immediately and investigate your claim 


\title{
Experimental vortex breakdown topology in a cylinder with a free surface
}

\author{
David Lo Jacono, ${ }^{1,2,3, a)}$ Mehdi Nazarinia, ${ }^{3}$ and Morten Brøns ${ }^{4}$ \\ ${ }^{1}$ INPT, UPS, IMFT (Institut de Mécanique des Fluides de Toulouse), Université de Toulouse, \\ Allée Camille Soula, F-31400 Toulouse, France \\ ${ }^{2}$ CNRS, IMFT, F-31400 Toulouse, France \\ ${ }^{3}$ Department of Mechanical and Aerospace Engineering, Fluids Laboratory for Aeronautical \\ and Industrial Research (FLAIR), Monash University, Melbourne, 3800 Victoria, Australia \\ ${ }^{4}$ Department of Mathematics, Technical University of Denmark, 2800 Kongens Lyngby, Denmark
}

(Received 21 July 2009; accepted 20 October 2009; published online 30 November 2009)

\begin{abstract}
The free surface flow in a circular cylinder driven by a rotating bottom disk is studied experimentally using particle image velocimetry. Results are compared with computational results assuming a stress-free surface. A dye visualization study by Spohn et al. ["Observations of vortex breakdown in an open cylindrical container with a rotating bottom," Exp. Fluids 14, 70 (1993)], as well as several numerical computations, has found a range of different vortex breakdown structures in this flow. We confirm the existence of a transition where the top of the breakdown bubble crosses from the axis to the surface, which has previously only been found numerically. We employ a technique by Brøns et al. ["Topology of vortex breakdown bubbles in a cylinder with rotating bottom and free surface," J. Fluid Mech. 428, 133 (2001)] to find the corresponding bifurcation curve in the parameter plane, which has hitherto only been used on numerical data. The bifurcation curve located here agrees well with previous numerical simulations. For low values of the Reynolds number we find a regime with vortex breakdown that has not been previously identified. Experiments deviate substantially from computations, indicating the importance of surface effects in this regime. (0) 2009 American Institute of Physics. [doi:10.1063/1.3265718]
\end{abstract}

The flow in a circular cylinder driven by a rotating bottom disk has proven to be a most useful setup to study secondary structures on a main vortex. Both experimental and computational studies have shown that one or more vortex breakdown zones or bubbles may occur in this flow. We refer the reader to previous reviews for a summary of the central features of vortex breakdown. ${ }^{1,2}$ In this letter we report experimental and computational results for the flow in a cylinder with a free surface. The first comprehensive experimental study of the closed cylindrical container case was undertaken by Escudier ${ }^{3}$ to obtain a map of vortex breakdown transitions with respect to aspect ratio and Reynolds number. Numerous computational studies $^{4-7}$ have reproduced the transitions very accurately. The first experimental study of the open cylindrical container case with a free surface on the top was undertaken by Spohn et al. ${ }^{8}$ Several computational studies of the flow assuming a stress-free, clean, and flat free surface are available. ${ }^{5,9-12}$ Comparing numerical and experimental results ${ }^{11}$ shows discrepancies not present in the closed cylinder case. Several flow topologies which are predicted numerically are not found experimentally, and the quantitative agreement between the numerical and computational results is generally poorer. It appears that physical effects such as surface deformation and surface contamination not included in this simple model influences the flow structure. Progress in the modeling of the flow has recently been obtained by Bouffanais and Lo Jacono ${ }^{13,14}$ who presented the results of a full numerical simulation in which the laminar, unsteady, and transitional flow regimes have been modeled without resort-

\footnotetext{
${ }^{a)}$ Electronic mail: david.lojacono@imft.fr.
}

ing to any symmetry property. Still, many challenges remain in modeling free surface flows ${ }^{15}$ making experimental studies of this problem especially important. There is currently a technological interest in the cylinder flow with a free surface as it provides a promising model for bioreactors. The steady flow within a vortex breakdown zone has a low stress level, making it appropriate for growth of organic tissue. ${ }^{16-18} \mathrm{Un}$ derstanding the topology of the flow-the number and shape of the breakdown bubbles - as it depends on the system parameters clearly becomes important.

In the present letter we examine the topology of vortex breakdown flow experimentally in a cylinder with a free surface using the particle image velocimetry (PIV) technique. We compare the results with numerical simulations assuming a flat stress-free surface. In the parameter regime we explore there is a unique, steady stable flow. It is the variation in the structures in this flow we explore.

The experimental setup consists of a transparent cylinder of diameter $D=2 R=65 \mathrm{~mm}$ that was filled with water and placed in the center of a water filled octagonal shaped container. The octagonal shape allows the exterior faces of the rig to be flat in order to reduce refraction effects. A similar experimental setup was successfully used elsewhere. ${ }^{19,20}$ The temperature of the water in the surrounding tank was controlled and kept at $20^{\circ} \mathrm{C}$ (Huber ministat) with a resolution of $0.1{ }^{\circ} \mathrm{C}$. The rotating flat circular disk was located at the bottom of the device. The shaft of this rotating plate was connected through a gear box (gear ratio of 40:1) to a high resolution stepper motor. The stepper motor was computer controlled via a high-performance motion controller at a resolution of 51200 steps rev $^{-1}$ (National Instrument MID- 
7604). The tracer particles used were spherical granular shaped polyamide particles with a nominal diameter of $20 \mu \mathrm{m}$ and specific weight of $1.016 \mathrm{gr} \mathrm{cm}^{-3}$. The particles were illuminated by a $2.0 \mathrm{~mm}$ thick laser sheet powered by two miniature Nd:yttrium aluminum garnet lasers (Continuum Minilite II) at a wavelength of $532 \mathrm{~nm}$ and maximum energy output of $25 \mathrm{~mJ}$ pulse ${ }^{-1}$. Pairs of images were captured on a high resolution camera with a maximum resolution of $4008 \times 2672$ pixel. The camera was equipped with a $200 \mathrm{~mm}$ lens. This setup gave a range of field of view which varied between $2672 \times 2170$ and $2672 \times 3800$ pixel $(1.232 \times 1.000 R$ and $1.232 \times 1.750 R$, respectively) for an aspect ratio of $\Gamma=H / R=1$ and $\Gamma=1.75$, respectively. The accuracy of the aspect ratio was estimated to be about 3 pixel at each end, equating to $1.38 \times 10^{-3} R$ at each end. It is worth noting that special care was taken to align the objectives, laser sheet, and camera, as any slight misalignment produces a non-negligible bias in the results. Each image pair was processed using in-house PIV software. ${ }^{19} \mathrm{~A}$ final interrogation window of $32 \times 32$ pixel $(0.48 \times 0.48 \mathrm{~mm}$ corresponding to $1.475 \times 1.475 \times 10^{-2} R$ ) was found to give satisfactory results with a $50 \%$ overlap (grid spacing of $16 \times 16$ pixels corresponding to $\left.7.375 \times 7.375 \times 10^{-3} R\right)$. Thus, the maximum measurement resolution obtained was $236 \times 166$ vectors for each field. A total number of 50 image pairs, taken at a $1.5 \mathrm{~Hz}$, were acquired. Measurements were started after $100-150$ cycles after the beginning of motion, allowing sufficient time to achieve steady state. These states display a high degree of symmetry which allows the determination of the axis of rotation. For a horizontal line at height $z$ which is parametrized by a variable $x$ we determine the point of intersection with the axis as the value of $x$ which minimizes the following measure of asymmetry:

$$
D(x)=\int[w(x+\xi)-w(x-\xi)]^{2} d \xi
$$

For a large region in the middle of the container this value of $x$ is well defined and is independent of $z$ and hence identifies the axis.

As the main quantity to identify changes in flow topology we use the axial velocity at the axis $w_{0}(z)$. We use dimensionless variables where the velocity is scaled by $R \Omega$, where $\Omega$ is the angular velocity of the bottom disk. The distance is scaled by $R$ such that $z$ varies from 0 at the bottom to $\Gamma$ at the free surface. Furthermore, we show streamlines of the flow from the isocurves of the streamfunction $\psi$ satisfying

$$
u=\frac{1}{r} \frac{\partial \psi}{\partial z}, \quad w=-\frac{1}{r} \frac{\partial \psi}{\partial r} .
$$

We find $\psi$ by integrating the second equation radially and choosing the level $\psi=0$ for the axis and the free surface. This method avoids using the radial velocity which is very small close to the axis and has a high relative uncertainty here. Away from the axis we have checked $\psi$ by comparing the measured $u$ with the one computed from Eq. (2) and found good agreement.
The incompressible Navier-Stokes equations were solved numerically using a spectral-element technique. The discretization method employed used seventh-order Lagrange polynomials associated with Gauss-LobattoLegendre quadrature points. The simulations were undertaken on a 400 macroelement mesh carefully concentrated toward the solid boundaries. Details of the implementation used here can be found in Ref. 21. The code employed has been well proven for use in bluff-body problems. ${ }^{22,23} \mathrm{Axi}$ symmetry and no-slip boundary conditions were applied on the appropriate sides of the computational domain. ${ }^{20}$

Figure 1 shows a typical sequence of flow structures for fixed $\Gamma$ and increasing Re. A corner bubble is present if $w_{0}<0$ in a region just below the surface. The bubble attaches to the axis at the point where $w_{0}=0$. In all cases there is good agreement between experiments and computations in the lower part of the cylinder, in particular, for the Ekman layer close to the rotating disk. Close to the free surface there are some discrepancies between experiments and computations, most prominently at the Re close to the corner crossing bifurcation. Experimentally, the flow topology can be clearly identified except for $\operatorname{Re}=1850$ where the sign of $w_{0}$ just below the surface cannot be determined unambiguously. Figure 2 summarizes our results for the corner crossing bifurcation. The existence of the bifurcation is clearly verified experimentally, and the precise location of it is in good agreement with computations, the maximal difference being around 100 in $R e$ at the highest values of $\Gamma$, with better agreement at lower $\Gamma$. From the experimental data an estimate of the bifurcation curve can be found as follows. Let $G(\Gamma, \mathrm{Re})$ $=w_{0}^{\prime}(0)$, i.e., the axial derivative of the axial velocity evaluated at the axis on the free surface. If $G>0$ a corner bubble is present, if $G<0$ it is not, see Fig. 1. Hence, the bifurcation can be located by the condition $G=0$. From the experimental data a series of values of $G$ can be obtained and a bifurcation function $\bar{G}$ is found by fitting these numbers using a secondorder polynomial in $\Gamma$ and Re. The bifurcation line $\bar{G}=0$ is shown as the dashed line in Fig. 2. The same procedure was used on numerical data. ${ }^{6}$ While it is to be expected that numerical data will depend smoothly on parameters such that the procedure will result in unambiguous results, it is less evident that the same holds for experimental data. However, this is the case for the present dataset. The bifurcation function $\bar{G}$ can be generated from a subset of the available data, and it turns out that the bifurcation curve is quite robust with respect to that choice. In particular, one may avoid data very close to the bifurcation line where the axial velocities close to the surface are very small, and hence $\partial w / \partial z$ is poorly defined. In fact, for the curve in Fig. 2 we have only used the data from the maximal and the minimal Re at each $\Gamma$. We now turn to the flow at low Re. Both previous computations ${ }^{11}$ and experiments ${ }^{8}$ indicate that as Re is decreased the corner bubble turns into a detached bubble which then shrinks and disappears. Figure 3 shows, however, some quantitative differences in the location of the two curves. Our experiments reproduce the first experimental bifurcation curve with good agreement. For decreasing Re we do find that the detached bubble shrink but does not disappear com- 

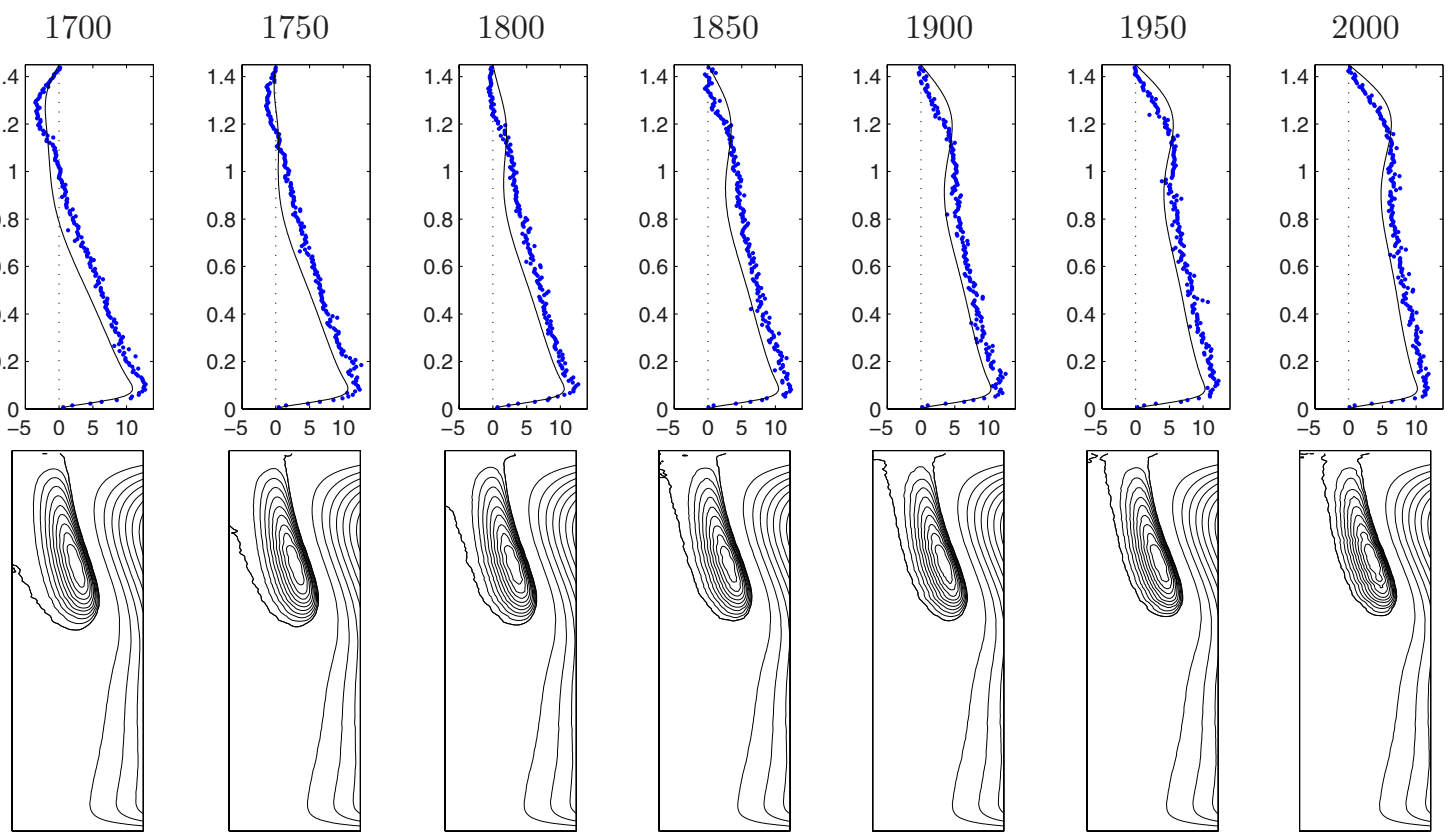

FIG. 1. (Color online) Transition from a corner bubble through a corner crossing bifurcation to an attached bubble at $\Gamma=1.45$ for increasing values of Re. The top row shows cylinder height vs $w_{0}(z) \times 10^{3}$. The markers are experimental results and the full curves are numerical results. Downward pointing velocities are positive. The bottom row shows corresponding isolines of $\psi$ obtained from the experimental data. The left edge is the cylinder axis, the right edge is located at $R / 2$.

pletely. At the same time the agreement with computations deteriorates, and at the lowest Re we find quite large breakdown bubbles with inner structures and an axial velocity profile which is completely different from the one obtained computationally, see Fig. 4. This behavior is consistent for all values of $\Gamma$. To the best of our knowledge, no experimental result in this regime is available in the literature. We attribute this discrepancy to surface effects which are not included in the computational model. We have taken great care to eliminate error sources such as vibrations and transients, but control of the surface properties is very difficult. Surface tension variations can give rise to stagnation points ${ }^{24}$ and the flow topology is consequently very sensitive to sur-

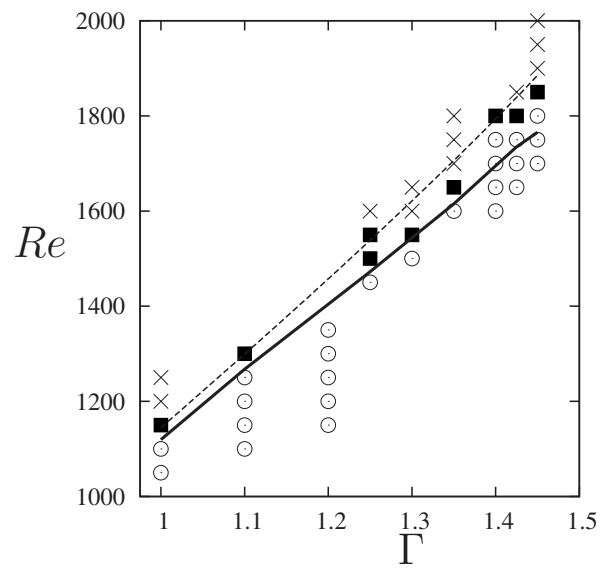

FIG. 2. Toplogical bifurcation diagram for the corner crossing bifurcation at high Re. The markers are experimental results. $\bigcirc$ : Corner bubble; $\times$ : attached bubble; $\mathbf{\square}$ : topology indeterminate. The full line is the numerically predicted corner crossing curve. The dashed line is the corner crossing curve determined from the experimental bifurcation function $\bar{G}$. face effects. This effect is dominating at low Re, since at high Re we get good agreement with computations assuming a stress-free surface.

In conclusion, we have shown that the present experimental technique allows a robust and reliable determination of vortex breakdown structures. We have confirmed the existence of a topological bifurcation where the topology changes from a corner bubble to an attached bubble through a corner crossing bifurcation that has previously only been found numerically. We have provided a proof of concept for a technique to determine a topological bifurcation curve

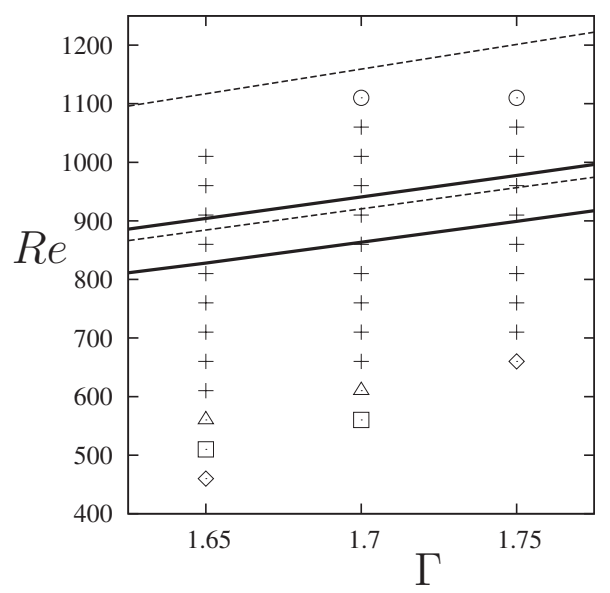

FIG. 3. Bifurcation diagram at low Re. The markers show experimental results. +: Detached bubble; $\bigcirc$ : corner bubble; $\times$ : attached bubble; $\triangle$ : detached+corner bubble; $\diamond$ : detached bubble with inner structure; $\square$ : corner bubble with inner structure. The full lines are bifurcation curves obtained from simulations. The lower curve is the creation of a detached bubble while the top curve is the corner crossing bifurcation to a corner bubble. The dashed lines are the corresponding experimental curves found by Spohn et al. (Ref. 8). 

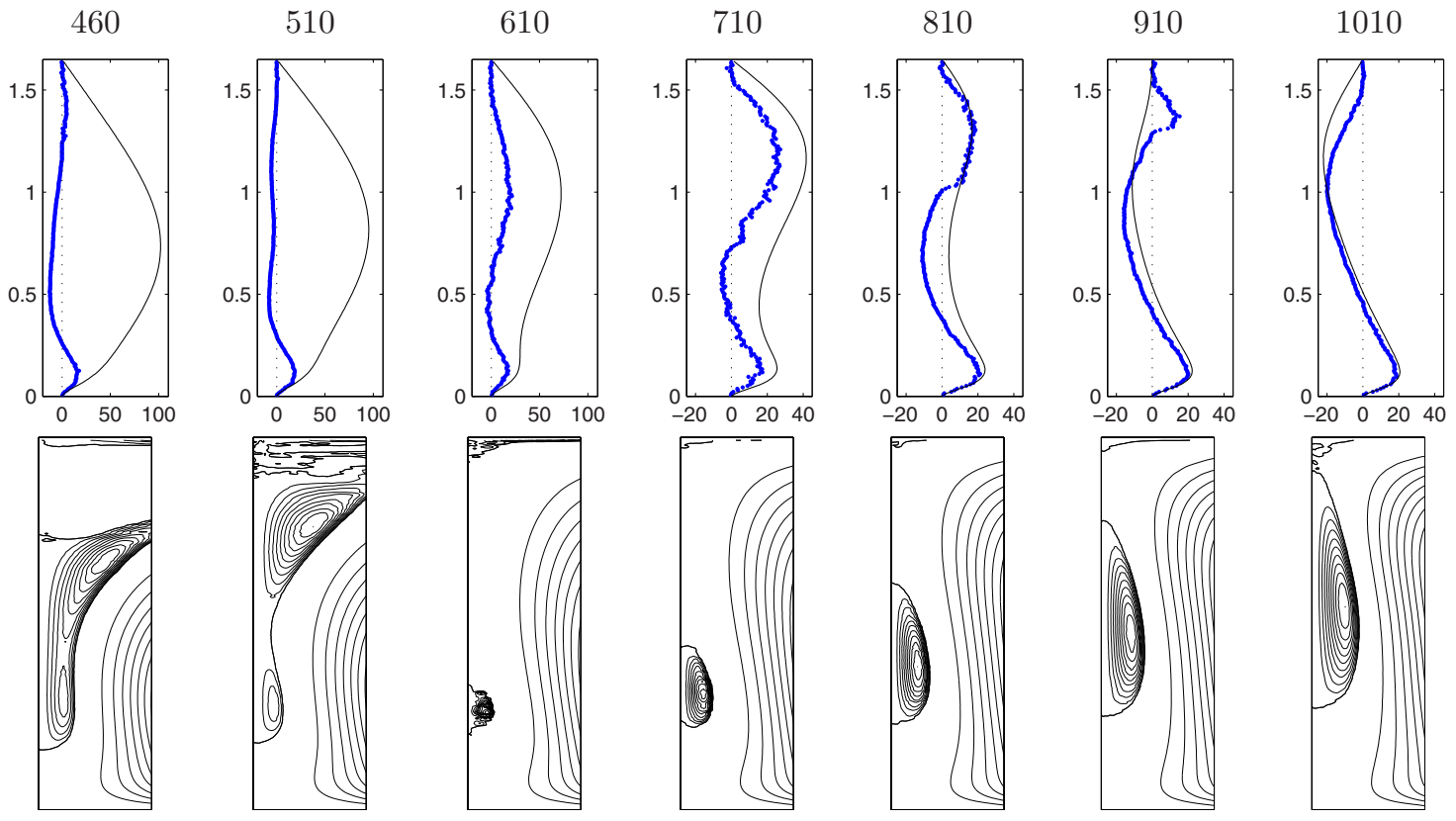

FIG. 4. (Color online) Flow topologies at $\Gamma=1.65$ and varying Re. Legend as in Fig. 1. Note the larger velocity range for the lowest Re.

from the quantitative data obtained experimentally. Finally, we have explored a new region of the parameter space where we have found vortex breakdown bubbles hitherto not observed nor found computationally. The existence of these bubbles indicate that surface effects are very important for the overall flow at low Re.

Parts of this research was conducted while M.B. was on a sabbatical leave at Monash University. The hospitality and excellent working conditions provided are gratefully acknowledged. M.N. would like to acknowledge the support of a Monash Graduate Scholarship MGS and a Monash International Postgraduate Research Scholarship MIPRS. D.L.J. acknowledges support from ARC Discovery (DP0774525) as well as Swiss National Science Fellowships (PBEL2-112259).

${ }^{1}$ M. G. Hall, "Vortex breakdown," Annu. Rev. Fluid Mech. 4, 195 (1972).

${ }^{2}$ S. Leibovich, "Vortex breakdown," Annu. Rev. Fluid Mech. 10, 185 (1978).

${ }^{3}$ M. P. Escudier, "Observations of the flow produced in a cylindrical container by a rotating endwall," Exp. Fluids 2, 189 (1984).

${ }^{4}$ J. M. Lopez, "Axisymmetric vortex breakdown Part 1. Confined swirling flow," J. Fluid Mech. 221, 533 (1990).

${ }^{5}$ A. Y. Gelfgat, P. Z. Bar-Yoseph, and A. Solan, "Steady states and oscillatory instability of swirling flow in a cylinder with rotating top and bottom," Phys. Fluids 8, 2614 (1996).

${ }^{6}$ M. Brøns, L. K. Voigt, and J. N. Sørensen, "Streamline topology of steady axisymmetric vortex break-down in a cylinder with co- and counterrotating end-covers," J. Fluid Mech. 401, 275 (1999).

${ }^{7} \mathrm{M}$. Brøns and A. V. Bisgaard, "Bifurcation of vortex breakdown patterns in a circular cylinder with two rotating covers," J. Fluid Mech. 568, 329 (2006).

${ }^{8}$ A. Spohn, M. Mory, and E. J. Hopfinger, "Observations of vortex breakdown in an open cylindrical container with a rotating bottom," Exp. Fluids 14, 70 (1993).

${ }^{9}$ D. T. Valentine and C. C. Jahnke, "Flows induced in a cylinder with both end walls rotating," Phys. Fluids 6, 2702 (1994).
${ }^{10}$ J. M. Lopez, "Unsteady swirling flow in an enclosed cylinder with reflectional symmetry," Phys. Fluids 7, 2700 (1995).

${ }^{11} \mathrm{M}$. Brøns, L. K. Voigt, and J. N. Sørensen, "Topology of vortex breakdown bubbles in a cylinder with rotating bottom and free surface," J. Fluid Mech. 428, 133 (2001).

${ }^{12} \mathrm{R}$. Iwatsu, "Numerical study of flows in a cylindrical container with rotating bottom and top flat free surface," J. Phys. Soc. Jpn. 74, 333 (2005).

${ }^{13}$ R. Bouffanais and D. Lo Jacono, "Transitional cylindrical swirling flow in presence of a flat free surface," Comput. Fluids 38, 1651 (2009).

${ }^{14} \mathrm{R}$. Bouffanais and D. Lo Jacono, "Unsteady transitional cylindrical swirling flow in presence of a moving free surface," Phys. Fluids 21, 064107 (2009).

${ }^{15}$ T. Sarpkaya, "Vorticity, free surface, and surfactants," Annu. Rev. Fluid Mech. 28, 83 (1996).

${ }^{16} \mathrm{P}$. Yu, T. S. Lee, Y. Zeng, and H. T. Low, "Effect of vortex breakdown on mass transfer in a cell culture bioreactor," Mod. Phys. Lett. B 19, 1543 (2005).

${ }^{17}$ J. Dusting, J. Sheridan, and K. Hourigan, "A fluid dynamics approach to bioreactor design for cell and tissue culture," Biotechnol. Bioeng. 94, 1196 (2006).

${ }^{18}$ Y. D. Cui, J. M. Lopez, T. T. Lim, and F. Marques, "Harmonically forced swirling flow," Phys. Fluids 21, 034106 (2009).

${ }^{19}$ A. Fouras, D. Lo Jacono, and K. Hourigan, "Stereo PIV: A novel technique with inherent error estimation and improved accuracy," Exp. Fluids 44, 317 (2008).

${ }^{20}$ D. Lo Jacono, J. N. Sørensen, M. C. Thompson, and K. Hourigan, "Control of vortex breakdown in a closed cylinder with a small rotating rod," J. Fluids Struct. 24, 1278 (2008).

${ }^{21}$ M. C. Thompson, K. Hourigan, and J. Sheridan, "Three-dimensional instabilities in the wake of a circular cylinder," Exp. Therm. Fluid Sci. 12, 190 (1996).

${ }^{22}$ M. Nazarinia, D. Lo Jacono, M. C. Thompson, and J. Sheridan, "Flow behind a cylinder forced by a combination of oscillatory translational and rotational motions," Phys. Fluids 21, 051701 (2009).

${ }^{23}$ M. Nazarinia, D. Lo Jacono, M. C. Thompson, and J. Sheridan, "The three-dimensional wake of a cylinder undergoing a combination of translational and rotational oscillation in a quiescent fluid," Phys. Fluids 21, 064101 (2009).

${ }^{24}$ M. Brøns, "Topological fluid dynamics of interfacial flows," Phys. Fluids 6, 2730 (1994). 